Rev Cuid. 2020; 11(1): e885 Open Access $\quad$ ARTÍCULO DE REVISIÓN

\title{
Soporte social y sobrecarga en cuidadores: revisión integrativa
}

\section{Social support and caregiver burden: an integrative review}

\author{
Suporte social e sobrecarga do cuidador: revisão integrativa
}

\section{Lorena Rodríguez-Lombana ${ }^{1}$, Lorena Chaparro-Diaz²}

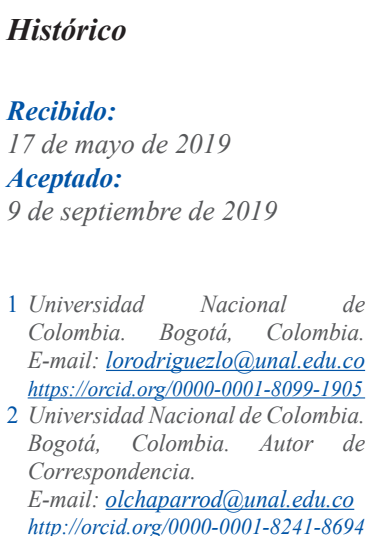

Introducción: Describir las intervenciones de soporte social dirigidas a cuidadores familiares de personas con Enfermedad Crónica No Transmisible que modifican la percepción de sobrecarga, a través de una revisión integrativa. Materiales y Métodos: Revisión integrativa de la literatura, con alcance descriptivo con la búsqueda de artículos correlacionados al fenómeno en los últimos 10 años y los cuales fueron potencialmente relevantes para identificar, evaluar e integrar las conclusiones sobre las intervenciones en soporte social que modifican la sobrecarga del cuidado. Rigor metodológico con los parámetros establecidos por Whittemore y Knafl, desarrollando la presentación de los datos con la Declaración Prisma. Resultados: Se observa el prisma del soporte social en los cuidadores familiares de personas con Enfermedad Crónica No Trasmisible; muestra de análisis de 19 piezas investigativas ( 9 artículos cuantitativos, 7 cualitativos y 3 mixtos) con abordaje del 100\% de la disciplina de enfermería. Análisis a partir de las categorías del perfil del cuidador, sobrecarga (física y emocional), cuidado de enfermería (a través del soporte social: informacional, emocional y cuidado continuo) y las intervenciones en soporte social, que se clasificaron desde lo educativo y emocional. Se identificó un vacío en el soporte instrumental ya que no se encontraron resultados asociados a este componente en las intervenciones de enfermería. Discusión: La mayoría de los estudios permitieron identificar que la mayoría de las intervenciones fueron individuales, grupales/familiares o la combinación de grupo familiar e individual. Conclusiones: Las intervenciones en soporte social a cuidadores familiares tienen una influencia positiva en la disminución de la sobrecarga del cuidado; se necesitan intervenciones a nivel rural y que aborden el soporte instrumental.

Palabras clave: Cuidadores; Apoyo Social; Enfermedad Crónica.

Abstract

Introduction: Through an integrative review, this study aims to describe the social support interventions on family caregivers of patients with Chronic Non-Communicable Diseases that modify the perception of caregiver burden. Materials and Methods: An integrative descriptive review of the literature was conducted for the search of articles correlated to the phenomenon over the last 10 years, which were potentially relevant to identify, evaluate and integrate the conclusions on social support interventions that modify caregiver burden. Methodological rigor was ensured following the parameters established by Whittemore and Knafl for data submission under the PRISMA Statement. Results: The prism of social support is observed in family caregivers of patients with Chronic Non-Transmissible Diseases with a sample of 19 pieces of research analyzed ( 9 quantitative articles, 7 qualitative articles, and 3 mixed articles), following a 100\% nursing approach. An analysis was made based on the categories of caregivers' profile, burden (physical and emotional), nursing care (through social support: informational, emotional and continuous care) and social support interventions ranging from educational to emotional aspects. A gap in instrumental support was found as we did not find any outcomes associated with this component in nursing interventions. Discussion: Most studies identified that most interventions were individual, group/family or the combination of family group and individual. Conclusions: Social support interventions on family caregivers have a positive influence on reducing caregiver burden. Interventions at the rural level are also required to address instrumental support.

Key words: Caregivers; Social Support; Chronic Disease.

Resumo

Introdução: Descrever as intervenções de apoio social direcionadas aos cuidadores familiares de pessoas com Doença Crônica Não Transmissível que modificam a percepção de sobrecarga, por meio de uma revisão integrativa. Materiais e Métodos: Revisão integrativa da literatura, com escopo descritivo, com busca de artigos correlacionados ao fenômeno nos últimos 10 anos e potencialmente relevantes para identificar, avaliar e integrar as conclusões sobre intervenções de apoio social que modificam a sobrecarga do cuidado. Rigor metodológico com os parâmetros estabelecidos por Whittemore e Knafl, desenvolvendo a apresentação dos dados com a Declaração Prisma. Resultados: O prisma do apoio social é observado em cuidadores familiares de pessoas com Doença Crônica Não Transmissível; amostra de análise de 19 trabalhos de pesquisa (9 quantitativos, 7 qualitativos e 3 artigos mistos) com abordagem $100 \%$ da disciplina de enfermagem. Análise baseada nas categorias de perfil do cuidador, sobrecarga (física e emocional), assistência de enfermagem (por meio de suporte social: assistência informacional, emocional e contínua) e intervenções de apoio social, classificadas nas categorias educacional e emocional. Foi identificada uma lacuna no suporte instrumental, pois não foram encontrados resultados associados a esse componente nas intervenções de enfermagem. Discussão: A maioria dos estudos permitiu identificar que a maioria das intervenções era individual, grupo / família ou a combinação de família e grupo individual. Conclusões: Intervenções de apoio social para cuidadores familiares influenciam positivamente na redução da sobrecarga de cuidado; são necessárias intervenções no nível rural e que abordem o apoio instrumental.

Palavras chave: Cuidadores; Apoio Social; Doença Crônica.

Cómo citar este artículo: Rodríguez-Lombana L, Chaparro-Diaz L. Soporte social y sobrecarga en cuidadores: Revisión integrativa. Rev Cuid. 2020; 11(1): e885. http://dx.doi.org/10.15649/cuidarte.885

(c) (i) (C) 2020 Universidad de Santander. Este es un artículo de acceso abierto, distribuido bajo los términos de la licencia Creative Commons Attribution (CC BY-NC 4.0), que permite el uso ilimitado, distribución y reproducción en cualquier medio, siempre que el autor original y la fuente sean debidamente citados. 


\section{INTRODUCCIÓN}

La Organización Mundial de la Salud define las Enfermedades Crónicas No Trasmisibles (ECNT) como una condición de salud que genera una alteración del funcionamiento orgánico o funcional permanente durante un periodo de tiempo mínimo de 6 meses. Su naturaleza es multicausal y no contagiosa, generalmente percibida por la persona que la padece como amenazante o incapacitante ${ }^{1}$.

En Colombia, se calcula son la causa del $71 \%$ del total de muertes registradas, con el $49.30 \%$ relacionadas con el sistema circulatorio, incluyendo las enfermedades cerebrovasculares, enfermedades hipertensivas y las enfermedades isquémicas del corazón. En segundo lugar, las neoplasias con un $15.03 \%$ siendo los tumores malignos de órganos digestivos y peritoneo los registrados como segunda causa de mortalidad $²$. Este panorama ha llevado en los últimos años a desarrollar diferentes políticas y programas de promoción y prevención que contribuyan al control, diagnóstico oportuno y manejo adecuado de las ECNT, como respuesta al aumento en los datos estadísticos asociados a la mortalidad.

Dentro de este proceso de cronicidad de una persona con ECNT, se experimenta necesidad de cambio de los estilos de vida, siendo un proceso que requiere supervisión, observación, cuidados especiales, adaptaciones y/o rehabilitación, por lo que toma posición de la persona en el contexto familiar, laboral y social ${ }^{3}$, lo que se expresa con sentimientos de pérdida de autonomía para el autocuidado, siendo necesario el acompañamiento, compromiso, apoyo y ayuda de otros constantemente, para lograr la adherencia al tratamiento y sobrellevar las exigencias especiales que la enfermedad trae consigo ${ }^{4}$.

Es así, que el cuidador familiar tomando este rol, se enfrenta a cambios en todos los aspectos de su vida, no solo al aceptar que su ser querido está viviendo en una situación de cronicidad, sino a la angustia del nuevo rol que debe asumir. Lo anterior, es producto de la exigencia en las actividades del cuidar relacionadas con la higiene, la alimentación, los medicamentos, las citas de control y el confort, viéndose afectada su forma independiente de vida, la libertad y la comodidad. Las investigaciones han demostrado consistentemente que los cuidadores experimentan enfermedades típicamente asociadas al estrés por la sobrecarga de cuidado como la depresión, ansiedad, fatiga, el aislamiento social, tensiones en las relaciones, disminución de la felicidad y tensiones financieras; en la salud física las manifestaciones son la reactividad cardiovascular, la disminución en la respuesta inmune, deterioro en su autocuidado con expresiones como el olvido en la toma de medicamentos, el cuidado en la dieta, ejercicio y sueño. Todo esto conlleva a la disminución de la calidad de vida y afectan la salud de los cuidadores $^{3-6}$. Sin embargo, también se pueden identificar aspectos positivos en esta labor, como el complemento para el propósito de la vida y la fortaleza interna, la formación de un "vinculo especial" ? la mayor cercanía con su familiar y mejorar el nivel de resiliencia emocional $\stackrel{8.9}{ }$. Por tal razón, las mediciones de la sobrecarga (tanto objetivas como subjetivas) dentro del rol de cuidador, son necesarias para determinar qué tanto se ve afectada su calidad de vida. 
Diferentes investigaciones realizadas en el contexto nacional e internacional demuestran que no coinciden los resultados encontrados entre las dos variables de sobrecarga, especialmente porque la población de cuidadores es diversa, con particularidades culturales diferentes y se manifiesta la necesidad de generar especificidad en el desarrollo del instrumento según los criterios particulares de cada cuidador $\frac{10,11,20,12-19}{}$. Es necesario tener en cuenta otros factores como el tipo, la severidad y trayectoria de la enfermedad, lo que modifica la percepción de la sobrecarga e incluso el nivel de dependencia o condición crónica de la persona ${ }^{8}$.

Un recurso externo que se ha identificado como herramienta para abordar las necesidades del cuidador es el soporte social, el cual provee del apoyo necesario en momentos de crisis, y se expresa como una transacción interpersonal de tres aspectos que se ofrecen de una persona a otra: apoyo emocional que provee el sentimiento y estima, afecto, confianza, seguridad, cariño, empatía. Este tipo de apoyo es el que más se relaciona con la salud y la resiliencia en el individuo que lo recibe. El apoyo instrumental, provee ayuda tangible y material para la solución de un problema. Herramienta que ayuda a modificar la severidad de la enfermedad al solventar las necesidades básicas de quien se cuida. Y el apoyo informacional, implica las intervenciones de aconsejar, informar o guiar. Trae resultados positivos cuando se suministran en cantidades moderadas ${ }^{21}$.
Por lo expuesto anteriormente, se justifica la necesidad de realizar una descripción y análisis de la literatura por falta de evidencia científica de las intervenciones de soporte social que modifican la percepción de sobrecarga del cuidado de cuidadores familiares de personas con enfermedad crónica no trasmisible.

\section{MATERIALES Y MÉTODOS}

El presente estudio se realiza bajo la metodología de Revisión Integrativa de la Literatura, teniendo un alcance descriptivo, con la búsqueda de artículos correlacionados en los últimos 10 años, debido a que en este último periodo de tiempo, se ha evidenciado el análisis del fenómeno desde diferentes perspectivas, por lo cual se han abordado con estudios de metodología cuantitativa, cualitativa y mixtas, los cuales son potencialmente relevantes para identificar, evaluar e integrar los datos, las interpretaciones y las conclusiones sobre las intervenciones en soporte social que de alguna forma modifican las percepción de sobrecarga de los cuidadores de personas con ECNT.

La elaboración de esta investigación se fundamentó en los parámetros establecidos de Whittemore y Knafl 22 cumpliendo con el rigor metodológico de las investigaciones primarias con las siguientes cinco etapas: 1 . identificación del problema, 2. búsqueda de literatura, 3. evaluación de los datos, 4. análisis de los datos y 5 . presentación de resultados $\underline{23}$. 


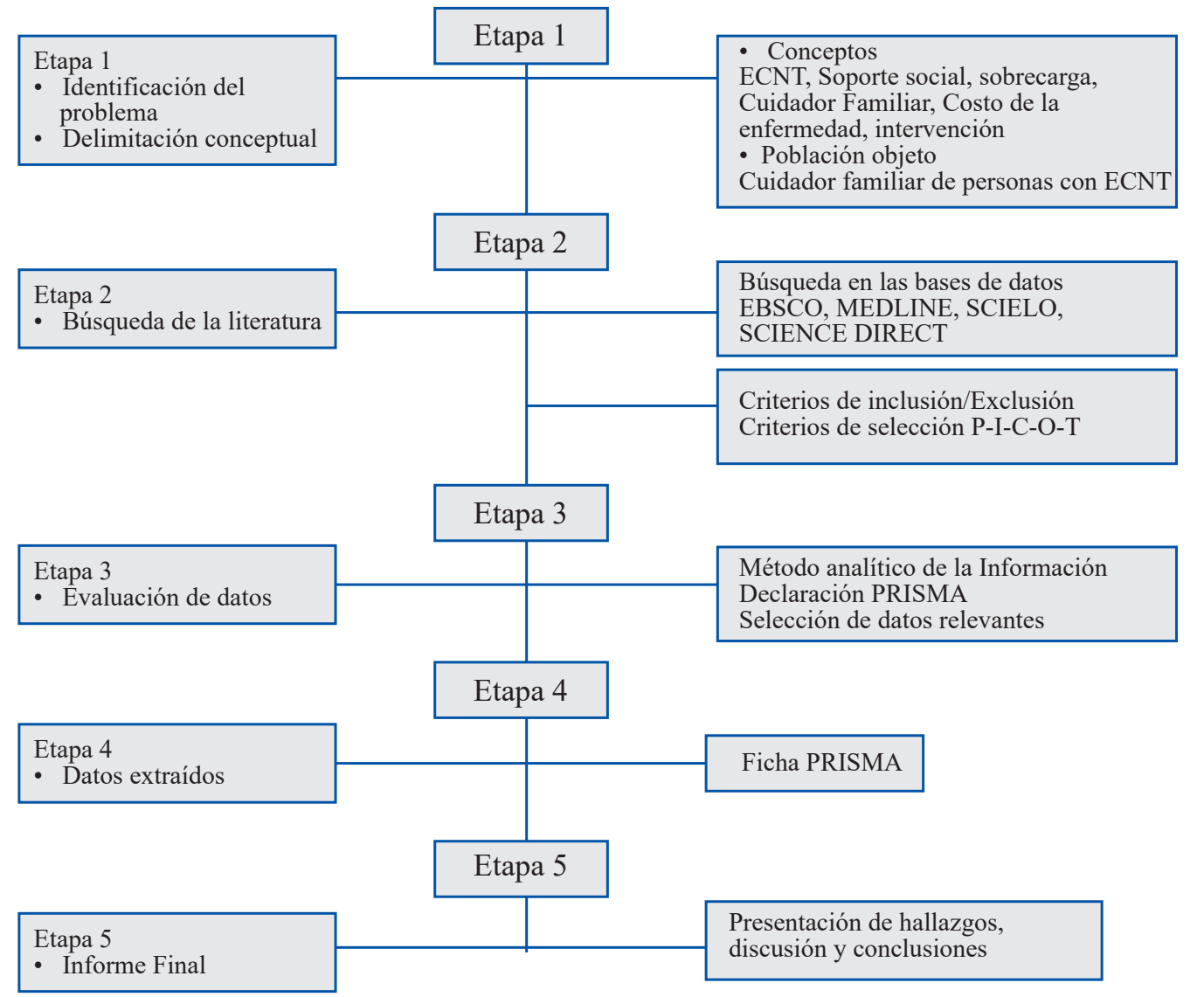

Figura 1. Proceso de selección de artículos

Fuente: Datos del estudio, 2017

En la Figura 1, se evidencia cada una de las etapas de rigor metodológico para revisiones integrativas propuesta por $\frac{23}{}$, y se correlaciona directamente con el cumplimiento en el desarrollo de la misma.

La primera etapa, se contó con los siguientes criterios de inclusión: artículos indexados, estudios cualitativos, cuantitativos y mixtos, en idioma inglés, español y portugués, en donde se realizó intervenciones en soporte social (emocional, informacional y emocional) con cuidadores familiares de personas con enfermedad crónica, que a su vez, modificaran la percepción de sobrecarga del cuidado; se desarrolló la pregunta PICOT, para definir los criterios de selección y se orienta la revisión, así:

P: Cuidadores familiares de personas con enfermedad crónica

I: Soporte social

C: Intervenciones

O: Sobrecarga

T: 2008-2018

Se excluyeron las investigaciones realizadas con madres gestantes, con enfermedades mentales crónicas, investigaciones realizadas con pacientes y no con cuidadores, por alejarse del fenómeno a investigar.

En la segunda etapa, se realizó la búsqueda de la literatura con los términos DECs/Mesh: enfer- 
medad crónica, soporte social, sobrecarga/costo de la enfermedad, cuidador familiar e intervención. Para las bases de datos de medline complete y EBSCO se utilizó la ecuación de búsqueda cuidador familiar AND apoyo social AND costo de la enfermedad / caregivers AND social support AND cost of illnes; para las bases de datos de Scielo y Science Direct se utilizó la ecuación cuidador familiar AND apoyo social / caregivers AND social support.

La presente investigación contó con 2 sesgos: Sesgos de selección, aplicado a la selección de bases de datos de mayor indexación en enfermería y ciencias de la salud, con una búsqueda exhaustiva para fundamentar el problema de in- vestigación con información veraz, acuciosa y precisa. Y el sesgo de desgaste, aplicado en que todos los artículos seleccionados se vieron reflejados en los resultados y se incluyeron en el análisis para las conclusiones del estudio.

En la etapa 3, la evaluación de los datos se realizó bajo un método analítico sistemático que permitiera la interpretación exhaustiva e imparcial de los datos de fuentes primarias, con ayuda de un segundo revisor de manera independiente, con el fin de evaluar la evidencia de las piezas investigativas para luego organizarlas según las intervenciones. En esta etapa se materializa la presentación de la Declaración Prisma, la cual se presenta a continuación:

\section{Infografía sobre las bases de datos}

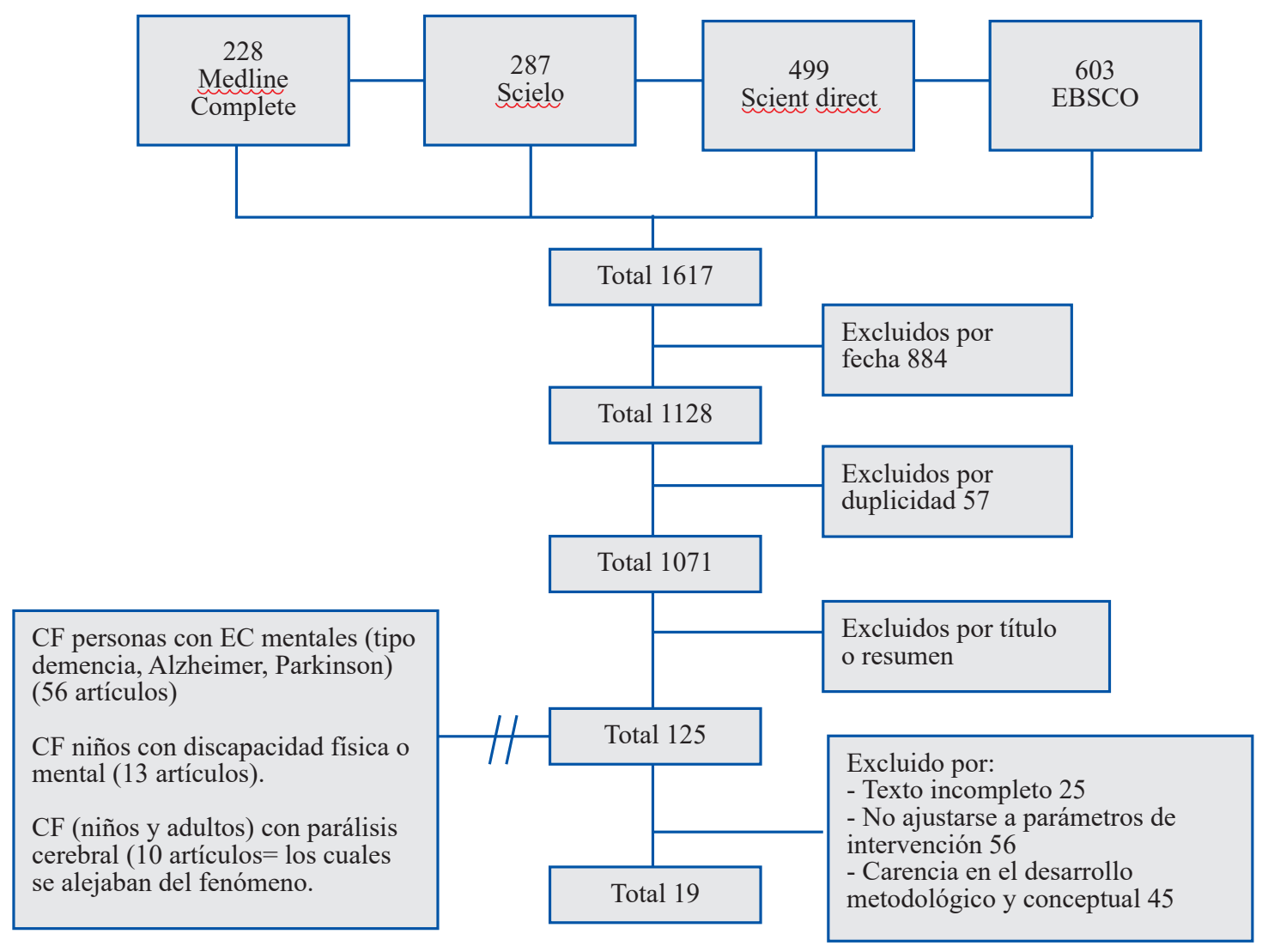

Figura 2. Declaración de la presentación PRISMA 
En la etapa 4, se tomaron los 19 artículos con un formato dispuesto para organizar los datos de tal manera que se pudieran visualizar y comparar, a través de un formato diseñado para tal fin. Se realizaron las clasificaciones por categorías para los esquemas de análisis y extracción de conclusiones (Figura 2).

\section{RESULTADOS}

En la Tabla 1, se presentan los 19 artículos que cumplieron con los criterios de inclusión para la revisión integrativa, y como se van a nombrar a continuación para la comprensión del lector.

Tabla 1. Publicaciones Incluidas

\section{No Título en Español Citación Vancouver}

1 Resiliencia y carga en los cuidadores y adultos mayores: efectos moderadores y mediadores del apoyo social percibido.

Ong Hl, Vaingankar JA, Abdin E, Sanbasi-

La carga de atención y los niveles de apoyo social de los cuidadores de pacientes con enfermedad pulmonar obstructiva crónica.

3 El video como soporte social a cuidadores de personas con Enfermedad Crónica, Girardot 2010. vam R, Fauziana R, Tan ME, Chong SA, Goveas RR, Chiam PC, Subramaniam M.

Göriş S ; Klç Z Elmal F Tutar N Takc Ö

Rojas MV ; Cardozo SL

4 El apoyo social influye en la carga del Liang SY, Shieh SC, Tung HS cuidador familiar primario en pacientes taiwaneses con cáncer colorrectal.

5 Efecto del programa Plan de egreso en la díada persona con enfermedad crónica y sus cuidadores familiares.

6 Evaluación del efecto del sentido de coherencia en la carga percibida y en la adherencia a un programa psicoeducativo para cuidadores informales de personas dependientes.

7 Vivir con la enfermedad pulmonar obstructiva crónica avanzada: el impacto de la disnea en los pacientes y cuidadores.

8 Efecto programa de apoyo social: percepción de cuidadoras familiares de personas mayores afrocolombianas en Guapi, Cauca.

9 Soporte social percibido en cuidadores familiares de personas en tratamiento contra el cáncer.
Carrillo Gloria Mabel, Melo Melo Blanca Gregoria, Vargas Hernández Yamile, Alarcón Trujillo Diana Katherine

Batlle Amat, Oriol Turró Garriga, Sílvia Farrés Costa Albert, Pérez Terréc Pau

Gómez Batisteb Xavier, Plad Margarida, Martínez Muñoz Marisa, Blayb Carles, Vila Laura

Maluche Sánchez Alexandra, Vilma Florisa Velásquez G.

Puerto Pedraza Henry Mauricio 32 
10 Soporte social con herramienta edumática para cuidadores familiares de personas con enfermedad crónica.
Díaz Juan Carlos, Álvarez Maribel, Pinilla

Alarcón Daniel, Eslava Albarracín

Gonzalo
11 Programa de apoyo al trabajador-cuidador familiar: fenómeno social emergente.
Herrera López Luz María, Marisa Torres

Claudia, Alcayaga Rojas Hidalgo

Abreu Rodrigues Marcela, Fleury Seidl Eliane Maria. medad coronaria: efectos de la conducta conductual con un miembro de la familia.

13 Las TIC como un mecanismo de apoyo Lorena Chaparro Díaz social para cuidadores de familia de pacientes con enfermedad crónica: un estudio de caso

14 Soporte social con Tecnologías de la Información y la Comunicación a cuidadores. Una experiencia en Cartagena, Colombia.

15 Soporte social con el uso de TIC's para cuidadores familiares de personas con enfermedad crónica.
Flórez Torres Inna Elida, Montalvo Prieto

Amparo, Romero Messa Elizabeth
Barrera Ortiz Lucy, Carrillo González Gloria M., Chaparro Díaz Lorena, Pinto Afanador Natividad y Sánchez Herrera Beatriz
16 Intervención para mejorar el apoyo social y familiar para los cuidadores de pacientes dependientes: protocolo de estudio ICIAS.
Baena Esther, Rosell Murphy Magdalena, Bonet Simó Josep, Prieto Gemma, Bellerino Eva, Solé Francesc, Rubio Montserrat, Krier Ilona, Torres Pascuala, Mimoso Sonia

17 Tiempo para vivir y cuidar: una intervención para hacer que el descanso sea más efectivo para los cuidadores.

18 Eficacia y costo- efectividad de un programa de salida para reducir la carga y la depresión de los cuidadores entre los cuidadores de pacientes con cáncer.

19 Redes Sociales, sociales de apoyo, y la carga en las relaciones, y la mortalidad después del diagnóstico de cáncer de mama en la vida después del estudio Breast Cancer Epidemiology (LACE).
Caserta Michael S., Dale A. Lund, Rebecca

L. Utz, Whright Scorr D., Llanque Sarah

M., Herb Shon, Whitlatch Carol J., Montoro Rodriguez Julian.
Livingston M. Patricia, Osborne Richard, Botti Mari, Mihalopoulos Cathy, Sean McGuigan, Leila Heckel, Gunn Kate, Chirgwin Jacquie, Ashley M David, Williams Melinda

Kroenke H. Candyce, Quesenberry Charles, Kwan L. Kwan, Sweeney Carol, Castillo Adrienne, Caan Bette J.

Fuente: Datos del estudio, 2017 
En la etapa 5, se realizó la presentación de las piezas investigativas, a través de un informe final que evidencia que el $100 \%$ de las publicaciones son de la disciplina de enfermería, correlacionadas a las intervenciones en soporte social dirigidas a cuidadores familiares de personas con ECNT que modifican de una u otra forma la percepción de sobrecarga del cuidado, y donde el $60 \%$ equivale a la formación académica de maestría.

Los países con más aportes en el tema, son Colombia $(40 \% / 8)$ y España $(15 \% / 3)$, aunque el idioma de indexación fue inglés $(50 \% / 10)$. Dentro de la revisión integrativa se encontró piezas literarias con diferentes metodologías: cuantitativa $(9 / 47 \%)$, cualitativa $(7 / 39 \%)$ y mixta $(3 / 15 \%)$ lo que permitió un abordaje del tema desde diferentes perspectivas (Tabla 2$)$.

Se realizó la clasificación de las piezas literarias a partir de los 3 tipos de apoyo social. se encontró que $11(60 \%)$ de los artículos tienen como base el apoyo informacional y 8 (40\%) obedecen a artículos de apoyo emocional. En cuanto a los enfoques teóricos existen 6 líneas que permiten entender que la sobrecarga del cuidador y la intervención sobre ésta puede ser analizada desde diversos puntos de vista. Para el caso del enfoque de salud pública, se determina como las enfermedades crónicas afectan también al cuidador, empeorando la situación epidemiológica y generando un complejo circulo entre la diada paciente - cuidador. En el caso de la teoría del estrés se observa que los estudios revisan la salud mental del cuidador, como elemento para planear la intervención. En cuanto a la línea educativa, reúne trabajos más diversos, que se refieren a la parte informacional del apoyo a los cuidadores, a través de los métodos para la enseñanza en el manejo de pacientes crónicos. En esta parte encontramos trabajos desde el enfoque diferencial, preocupados por la condición étnica y otros desde la edumática.

\section{Se encontró que los instrumentos utilizados en} las piezas investigativas corresponden a:

- Para caracterización del cuidador, el más usado fue el de la UNAL propuesto por el GCPC

- Para medir el soporte social, el más usado fue el instrumento de Soporte Social Percibido propuesto por G. Hilbert, seguido, la Escala Multidimensional de Apoyo Social percibido de Zimet.

- Para medir la sobrecarga del cuidado, la Escala de Zarit. 
Tabla 2. Tipo de investigación y metodología utilizada por las diferentes piezas literarias

\begin{tabular}{lllc}
$\begin{array}{l}\text { Tipo de } \\
\text { investigación }\end{array}$ & Metodología & Número & Citación Vancouver \\
Cuantitativo & Estudio transversal & 2 & 26,27 \\
\cline { 2 - 4 } & Estadística descriptiva & 3 & $25,32,38$ \\
\cline { 2 - 4 } & Estudio cuasi-experimental & 2 & 28,31 \\
\cline { 2 - 4 } & Ensayo. Multicéntrico & 2 & 26,39 \\
\hline \multirow{2}{*}{ Cualitativo } & $\begin{array}{l}\text { Recopilación de Narrativas (a } \\
\text { través de entrevista semiestructu- } \\
\text { rada, historias de vida) }\end{array}$ & 2 & \\
\cline { 2 - 4 } & Perspectiva fenomenológica & 1 & 34 \\
\cline { 2 - 4 } & Investigación acción participativa & 1 & 30 \\
\cline { 2 - 4 } & Estudio Exploratorio-descriptivo & 2 & 36,33 \\
\cline { 2 - 4 } & Utilización de Escala de Zarit y & 1 & 41 \\
\hline \multirow{2}{*}{ Mixto } & Prospectivo Observacional & 2 & 42,29 \\
\hline & $\begin{array}{l}\text { Socio - educativo integra diarios } \\
\text { de campo y escala de Zarit }\end{array}$ & 1 & 24 \\
\hline
\end{tabular}

Fuente: Datos del estudio, 2017

Dentro de las intervenciones en soporte social planteadas en las piezas investigativas, se encontraron:

Tabla 3. Tipo de intervención realizada como soporte social a cuidadores familiares de personas con Enfermedad Crónica

\begin{tabular}{|c|c|c|}
\hline Intervención en soporte social & $\begin{array}{l}\text { Cantidad de artículos que } \\
\text { usaron esta metodología }\end{array}$ & Citación Vancouver \\
\hline $\begin{array}{l}\text { Entrega de cartillas, folletos e } \\
\text { instructivos }\end{array}$ & 2 & 40,31 \\
\hline $\begin{array}{l}\text { Llamadas telefónicas } \\
\text { (ayuda y/o seguimiento) }\end{array}$ & 1 & 33 \\
\hline $\begin{array}{l}\text { Grupos psicoeducativos } \\
\text { (sesiones educativas) }\end{array}$ & 6 & $24,25,29,32,38,39$ \\
\hline Programas de apoyo social & 3 & $33,38,39$ \\
\hline Radio & 1 & 37 \\
\hline Internet (redes sociales, blog) & 3 & $36,37,38$ \\
\hline DVD - CD & 3 & $33,26,38$ \\
\hline
\end{tabular}

Fuente: Datos del estudio, 2017 
Se evidencia que se necesita de un elemento físico acompañado de las sesiones educativas para que se encuentre adherencia de los cuidadores familiares a las intervenciones y realmente se impacte en la disminución de la sobrecarga en el cuidado.

\section{DISCUSIÓN}

Se realiza un análisis de los documentos encon- trados sobre las intervenciones en soporte social dirigidas a cuidadores familiares de personas con enfermedad crónica no transmisible que modifican la percepción de la sobrecarga, las cuales se analizan desde los dos tipos de apoyo social que se encontraron como herramienta de intervención: el apoyo informacional y el emocional.

En la Figura 3, se concentra la información a partir de 3 categorías, así:

\section{Intervenciones de Soporte Social que influyen en la sobrecarga de cuidadores familiares de personas con ECNT}

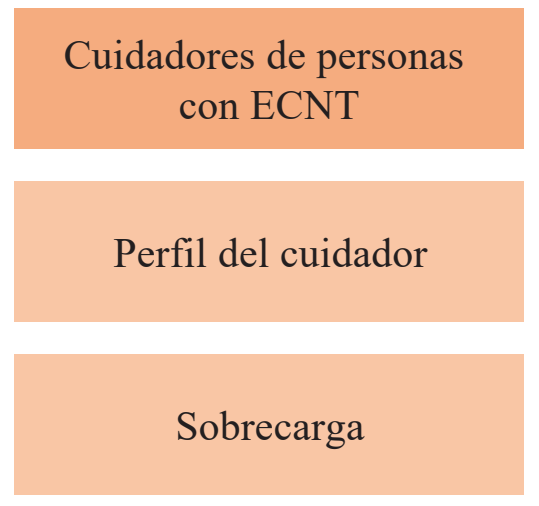

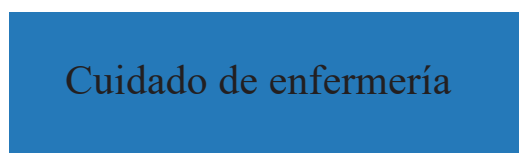

Apoyo informacional

Apoyo Emocional

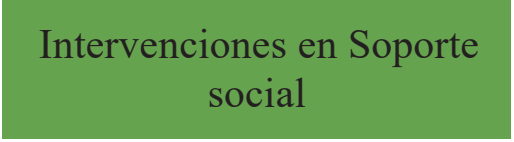

Desde lo educativo

Desde lo emocional

\section{Figura 3. Categorías de intervenciones en Soporte Social}

Fuente: Datos del estudio, 2017

Se aborda la primera categoría, de Cuidadores de pacientes con ECNT, donde se considera el perfil del cuidador, los cuales en su mayoría muestran a la mujer como la encargada de brindar el cuidado a pacientes en condición de cronicidad en el hogar $\frac{28-33}{3}$. Por tanto, la variable de género es fundamental para describir el elemento de la sobrecarga, en la medida que culturalmente se cree que el cuidado ha sido liderado por las mujeres. Existe una mediana de $52 \pm 16$ años y se indica que existe un parentesco en primer y segundo grado de consanguinidad, siendo las madres, las hijas, las esposas e incluso las nietas las encargadas del cuidado ${ }^{28-33}$.

Las razones para asumir el cuidado del paciente, se encuentra el de los costos del cuidado y la incapacidad del paciente para adaptarse a un cuidador externo $\frac{30.34}{}$, pero se observa también, que existe un envejecimiento en la población de cuidadores, lo cual hace que el cuidado sea mucho más complejo ${ }^{35}$. 
En la sobrecarga, se encontró que varios de los artículos cualitativos y cuantitativos recogen datos tales como el desarrollo de síntomas de depresión y de enfermedades coronarias asociados a sentimientos negativos y de frustración por el estado del paciente ${ }^{29,30}$. En este sentido la sobrecarga debe ser trabajada a través de planes de autocuidado emocional $29,30,27,33,40-42$. En cuanto a la sintomatología por sobrecarga física está asociado a aquellos cuidadores que perciben mayor dependencia por parte de los pacientes en sus tareas diarias $25,39,41$. Desde esta perspectiva existen variables que están asociadas a la funcionalidad de la relación de la díada paciente-cuidador. Siendo importante resaltar que en aquellos estudios donde el paciente coopera con el cuidado es menor la percepción de $\operatorname{carga}{ }^{25}$.

Desde el Cuidado de Enfermería, se realiza un análisis del papel de enfermería en este contexto, el cual está centrado en dirigir acciones de promoción de la salud y prevención de la enfermedad, desde el contexto del autocuidado. Se considera esencial establecer desde la literatura los elementos que ofrecen tanto el apoyo informacional como emocional, derivados de la intervención de enfermería, para posteriormente establecer la caracterización de un modelo de Cuidado Continuo que contribuya al apoyo instrumental y a disminuir los riesgos de presentar patologías agudas y cónicas, disminuyendo la sobrecarga del cuidado y aumentando la calidad de vida de la diada y la familia ${ }^{\underline{28}}$.

En cuanto a las intervenciones en soporte social, se presentan las particularidades encontradas a partir de las categorías educativas y psicosociales. Se encuentran tres modalidades de inter- vención: individuales, grupales/familiares o la combinación de grupo familiar e individual 24-26-33, $\underline{36,37,41}$.

\section{CONCLUSIONES}

Se concluye que la Revisión Integrativa de la literatura tiene un rigor metodológico exigente que permite observar el prisma del soporte social en los cuidadores familiares de personas con ECNT, con una muestra de análisis de 19 piezas investigativas con abordaje del $100 \%$ de la disciplina de enfermería.

Se identifica que las necesidades de los cuidadores, abarca tanto lo instrumental como los espacios de reflexión, interrelación y autocuidado, para el afrontamiento de su rol de cuidador. Se evidencia que cuando se cuenta con un soporte social efectivo hay una disminución de la sobrecarga y mejora el grado de funcionalidad de los hogares; o en el contexto inverso, si aumenta la sobrecarga, disminuye la funcionalidad familiar.

Se requiere de intervenciones educativas y psicosociales que se orienten con instrumentos de aprendizaje, con intervención del trabajo interdisciplinario con otras áreas para el fortalecimiento de la red de apoyo para la planificación de objetivos e intervenciones unificadas.

Se hace necesario el abordaje de políticas públicas que permitan incluir al cuidador y su rol dentro del sistema, para disminuir la sobrecarga del cuidado, siendo un elemento clave para el desarrollo de un modelo integral de atención en salud. 
Es necesario abordar el soporte social desde lo instrumental y contribuir con la producción de piezas investigativas para registrar el actuar diario de los profesionales en enfermería con la diada y su familia.

\section{Conflicto de intereses: Los autores declaran no}

tener conflicto de intereses.

\section{REFERENCIAS}

1. Organización Mundial de la Salud. Análisis de situación de salud. 2015 [cited 2017 Aug 23]; 240-2. Available from: https://www.minsalud.gov.co/sites/rid/Lists/BibliotecaDigital/RIDE/VS/ED/PSP/asis-2015.pdf

2. Ministerio de salud y protección social. Análisis de situación de salud (asis) Colombia, 2016 Dirección de Epidemiología y Demografía Bogotá, noviembre de 2016. 2016 [cited 2017 Aug 24]. https:/www.minsalud.gov.co/ sites/rid/Lists/BibliotecaDigital/RIDE/VS/ED/PSP/asiscolombia-2016.pdf

3. López-León D, Rodríguez-Calderón L, Carreño-Moreno S, Cuenca I, Chaparro-Díaz L. Cuidadores de pacientes en diálisis peritoneal: experiencia de participar en un programa de habilidad de cuidado. Enfermería Nefrológica. 2015; 18(3):189-95.

https://doi.org/10.4321/S2254-28842015000300007

4. Barrera L, Pinto N, Sánchez B, Carrillo GM, Chaparro L. Cuidando a los cuidadores familiares de personas con enfermedad crónica. 2010; Universidad Nacional de Colombia. 360 p.

5. Carreño SP, Chaparro-Díaz L, Patricia S, Lorena CY. Agrupaciones de cuidadores familiares en Colombia : perfil, habilidad de cuidado y sobrecarga. Pensamiento Psicológico. 2017;15(1):87-101.

6. Carrillo GM, Sánchez B, Barrera L. Habilidad de cuidado de cuidadores familiares de niños con cancer. Index de Enfermería. 2015; 23(3): 394-403.

https://doi.org/10.15446/rsap.v17n3.32408

7. Chaparro L. Cómo se constituye el "vínculo especial" de cuidado entre la persona con enfermedad crónica y el cuidador familiar. Aquichán. 2011; 11(1): 7-22. https://doi.org/10.5294/aqui.2011.11.1.1

8. Revenson TA, Konstadina G, Luszczynska A, Morrison V, Panagopoulou E, Vilchinsky N, et al. Caregiving in the Illness Context. 2016. Palgrave Pivot, London. 166 p. https://doi.org/10.1057/9781137558985

9. Braun M, Mikulincer M, Rydall A, Walsh A, Rodin G. Hidden morbidity in cancer: spouse caregivers. J Clin Oncol. 2007; 25(30): 4829-34.

https://doi.org/10.1200/JCO.2006.10.0909
10. Arias M, Barrera L, Carrillo GM, Chaparro L, Sánchez B, Vargas E. Cuidadores familiares de personas con enfermedad crónica en las regiones de frontera colombiana: perfil y carga percibida de cuidado. Rev la Fac Med. 2014- 10; 62(3):387-97.

https://doi.org/10.15446/revfacmed.v62n3.39091

11. Ballesteros JE, Rodríguez-Quenza AM, CantorChávez M, Peñalosa-González GC, Valcárcel DE. Caracterización y percepción de carga de cuidadores familiares de personas con enfermedad crónica en Arauca. Orinoquia. 2015; 19(1): 100-5. https://doi.org/10.22579/20112629.343

12. Barrera L, Carrillo GM, Chaparro L, Sánchez B. Modelo para abordar la carga del cuidado de la enfermedad crónica en Colombia. Orinoquia. 2015; 19(1): 89-99. https://doi.org/10.22579/20112629.342

13. Cárdenas-Corredor DC, Melenge-Díaz B, Pinilla J, Carrillo-González GM, Chaparro-Díaz L. Soporte social con el uso de las TIC para cuidadores de personas con enfermedad crónica: un estado del arte. Aquichan. 2010; 10(3): 2014-13.

14. Carrillo GM, Chaparro-Díaz L, Herrera BS. Carga del Cuidado en Cuidadores Familiares de Personas Con Enfermedad Crónica en la Región Pacífica Colombiana. Cienc. enferm. 2014; (2):83-91. https://doi.org/10.4067/S0717-95532014000200009

15. Goren A, Gilloteau I, Lees M, DaCosta DiBonaventura M. Quantifying the burden of informal caregiving for patients with cancer in Europe. Support Care Cancer. 2014; 22(6):1637-46. https://doi.org/10.1007/s00520-014-2122-6

16. Moreno SPC, Osorio RVB, Parra MD, Ortiz VT, Romero E. Habilidad de cuidado y sobrecarga en cuidadores familiares de personas con enfermedad crónica. $\mathrm{Rev} C u$ bana Enferm. 2016; 32(3).

17. Cordova FM, Harris RB, Teufel-Shone NI, Nisson PL, Joshweseoma L, Brown SR, et al. Caregiving on the Hopi Reservation: Findings from the 2012 Hopi Survey of Cancer and Chronic Disease. J Community Health. 2016; 41: 1177-86. https://doi.org/10.1007/s10900-016-0199-1

18. Kevser S, Dilek A. The Care Burden of Caregivers Caring for Elderly with Chronic Diseases and Affecting Factors. 2016;7: p1-9.

19. Göriş S, Klç Z, Elmal F, Tutar N, Takc Ö. Care Burden and Social Support Levels of Caregivers of Patients with Chronic Obstructive Pulmonary Disease. Holist Nurs Pract. 2016; 30(4):227-35. https://doi.org/10.1097/HNP.0000000000000153

20. Sulaiman AA, Abdullah AA, Khaled AA, Abdulaziz AA, Yazeed AA, Mohammed AK, et al. The burden perceived by informal caregivers of the elderly in Saudi Arabia. J Fam Community Med. 2017; 24:(3): 145-50.

21. Vega Angarita OM, González Escobar DS. Apoyo social: elemento clave en el afrontamiento de la enfermedad crónica. Enfermería Glob. 2009; (16). https://doi.org/10.4321/S1695-61412009000200021 
22. Whittemore R, Knafl K. The integrative review: Updated methodology. J Adv Nurs. 2005; 52(5): 546-53. https://doi.org/10.1111/j.1365-2648.2005.03621.x

23. Whittemore R. The integrative review: updated methodology. 2005; Oregon Health and Sciences University, Portland.

24. Ong HL, Vaingankar JA, Abdin E, Sambasivam R, Fauziana R, Tan ME, et al. Resilience and burden in caregivers of older adults: moderating and mediating effects of perceived social support. BMC Psychiatry. 2018; 18(1): 27. https://doi.org/10.1186/s12888-018-1616-Z

25. Songul G, Zuleyha K, Ferhan E, Nuri T, Ozlem T. Care Burden and Social Support Levels of Caregivers of Patients with Chronic Obstructive Pulmonary. Disease. 2016; 4: 227-35. https://doi.org/10.1097/HNP.0000000000000153

26. Rojas-Martinez MV, Cardozo-Silva SL. El video como soporte social a cuidadores de personas con enfermedad crónica, Girardot 2010. Av en Enferm. 2010; 33(2):199208. https://doi.org/10.15446/av.enferm.v33n2.49784

27. Shieh S-C, Tung H-S, Liang SY. Social Support as Influencing Primary Family Caregiver Burden in Taiwanese Patients With Colorectal Cancer. J Nurs Scholarsh. 2012; 44(3): 223-31. https://doi.org/10.1111/j.1547-5069.2012.01453.x

28. Melo BG, Vargas Y, Carrillo GM, Alarcón DK. Efecto del programa Plan de egreso en la díada persona con enfermedad crónica y sus cuidadores familiares. Enfermería Clínica. 2018; 28(1):36-43.

https://doi.org/10.1016/j.enfcle.2017.09.003

29. Turró O, Farrés S, Pérez A, Batlle P. Evaluación del efecto del sentido de coherencia en la carga percibida y en la adherencia a un programa psicoeducativo para cuidadores informales de personas dependientes. Rev Esp Geriatr Gerontol. 2018; 53(4):196-201.

https://doi.org/10.1016/j.regg.2017.12.002

30. Costa X, Gómez-Batiste X, Pla M, Martínez-Muñoz M, Blay C, Vila L. Atención Primaria Vivir con la enfermedad pulmonar obstructiva crónica avanzada: el impacto de la disnea en los pacientes y cuidadores. Atención Primaria. 2016; 48(10): 665-73. https://doi.org/10.1016/j. aprim.2016.02.008

31. Maluche-Sánchez A, Velásquez-G VF, Maluche-Sánchez A, Velásquez-G VF. Effect of a social support program: perception of family caregivers of afrocolombian elderly, Guapi - Cauca. Hacia la Promoción la Salud. 2017; 22(2): 99-110.

32. Puerto HM. Soporte social percibido en cuidadores familiares de personas en tratamiento contra el cáncer. Rev Cuid. 2017; 8(1): 1407-22. http://dx.doi.org/10.15649/cuidarte.v8i1.345

33. Díaz JC, Pinilla M, Eslava DG, Padilla MP. Soporte social con herramienta edumática para cuidadores familiares de personas con enfermedad crónica. Aquichan. 2014; 14(3):364-87.

https://doi.org/10.5294/aqui.2014.14.3.8
34. Herrera LM, Alcayaga C, Torres M, Funk R, Bustamante C, Riquelme G, et al. Programa de apoyo al trabajador-cuidador familiar: fenómeno social emergente. Aquichan. 2014; 14(3):430-9. https://doi.org/10.5294/aqui.2014.14.3.12

35. Abreu-Rodrigues M, Seidl EMF. Apoio social a pacientes coronarianos: efeitos de intervenção comportamental com um familiar. Estud Psicol. 2013; 30(4):619-28. https://doi.org/10.1590/S0103-166X2013000400015

36. Chaparro-Díaz L, Sánchez-Herrera B, Carrillo GM, Barrera-Ortiz L. Las TIC como un mecanismo de apoyo social para cuidadores de familia de pacientes con enfermedad crónica: Un estudio de caso. Aquichan. 2013;13(1):27-40.

37. Flórez IN, Montalvo A, Romero E. Soporte social con Tecnologías de la Información y la Comunicación a cuidadores: una experiencia en Cartagena, Colombia. Investig y Educ en enfermería. 2012; 30(1): 55-65.

38. Barrera-Ortiz L, Carrillo-González GM, ChaparroDíaz L, Afanador NP, Sánchez-Herrera B. Soporte social con el uso de TIC's para cuidadores familiares de personas con enfermedad crónica. Revista de Salud Pública. 2011; 13(3):446-57.

39. Rosell-Murphy M, Bonet-Simó JM, Baena E, Prieto G, Bellerino E, Solé F, et al. Intervention to improve social and family support for caregivers of dependent patients: ICIAS study protocol. BMC Fam Pract. 2014; 15(1):53. https://doi.org/10.1186/1471-2296-15-53

40. Lund DA, Utz RL, Caserta MS, Wright SD, Llanque SM, Lindfelt C, et al. Time for living and caring: an intervention to make respite more effective for caregivers. Int $J$ Aging Hum Dev. 2014; 79(2):157-78.

https://doi.org/10.2190/AG.79.2.d

41. Livingston PM, Osborne RH, Botti M, Mihalopoulos C, McGuigan S, Heckel L, et al. Efficacy and cost-effectiveness of an outcall program to reduce carer burden and depression among carers of cancer patients [PROTECT]: rationale and design of a randomized controlled trial. BMC Health Serv Res. 2014; 14(1):5.

https://doi.org/10.1186/1472-6963-14-5

42. Kroenke CH, Quesenberry C, Kwan ML, Sweeney C, Castillo A, Caan BJ. Social networks, social support, and burden in relationships, and mortality after breast cancer diagnosis in the Life After Breast Cancer Epidemiology (LACE) study. Breast Cancer Res Treat. 2013; 137(1):261-71. https://doi.org/10.1007/s10549-012-2253-8 